\title{
An Energy-Efficiency Protocol in Wireless Sensor Networks using Theory of Games and Fuzzy Logic
}

\author{
Mahnaz Esmaeeli \\ Department of computer, Dezful Branch, Islamic \\ Azad University, Dezful Iran
}

\author{
Seyed Ali Hosseini Ghahroudi \\ Professor Department of computer, Arsangan \\ Branch, Islamic Azad University, Arsangan Iran
}

\begin{abstract}
Today, cluster-based routing protocols achieve the highest efficiency in network life duration and network coverage- as compared with other routing methods- by dividing neighboring nodes into separate clusters and selecting local cluster heads for each node data combination and transmission to the basic station as well as by consuming energy in a balanced way. This study offers a centralized energy-based clustering protocol for WSNs on the basis of game theory and fuzzy logic in order to cluster network nodes by considering energy level and proximity of nodes. The simulation findings reveal the protocol's high efficiency in network life as compared with LEACH and other algorithms.
\end{abstract}

\section{Keywords}

Wireless Sensor Networks, Theory of Games, Fuzzy Logic, Clustering, Energy Consumption.

\section{INTRODUCTION}

One of crucial means for gathering information on environment and gaining an understanding of it, which has been investigated broadly, is Wireless Sensor Network (WSN). Today, the designed sensors consume low power, are small in size and are reasonably priced, and have a variety of uses. Accordingly, WSNs are created and developed. WSNs can contain hundreds or thousands of sensor nodes. Despite extensive studies on WSNs, sensor nodes are still dependent on small batteries due to their multiplicity and small size $[1 \&$ 2]. Additionally, since these networks are used in inaccessible and dangerous environments, network nodes cannot be chargedor replaced. Therefore, energy consumption should be optimized and network life should be prolonged.

These networks usually have a basic station, which is related with other sensors wirelessly. Each sensor sends its own data to the basic station directly or by the help of other nodes. Then, the collected information of the concerned parameter is processed in the basic station and its exact value is estimated with a relative precision. The clustering-based protocol is one of protocols for prolonging life nodes and reducing energy consumption. It reduces network energy consumption considerably. In this model, the network is divided into some clusters, each of which involves a number of member nodes and a cluster head [ $3 \& 4]$. Nodes directly send its data to the related cluster node which in turn sends the collected and combined data to the basic station. In the other words, each node does not directly send its data to the basic station. By choosing one node in each cluster as the cluster head and by aggregating multiple data, the network life is prolonged [1-6].
The present study attempts to offer an algorithm for reducing or minimizing energy consumption of WSNs relative to the other existing methods. This algorithm is clustering-based and is designed with regard to theory of games and fuzzy logic. In the second section, the conducted studies are evaluated. Then, the considered network model is explained. The proposed method and the simulation results are examined in sections 4 and 5 respectively. Finally, suggestions for further studies and the study conclusion are offered.

\section{REVIEW OF LITERATURE}

Low Energy Adaptive Clustering Hierarchy (LEACH) is the first and the most well-known clustering-based protocol in wireless sensor networks (WSNs). The most crucial aim of LEACH is the possession of local basic stations (cluster heads) for reducing energy consumption caused by transmission of data to a distant basic station. LEACH randomly selects a small number of cluster heads and organizes local nodes as local clusters [7]. Nodes are assigned to cluster heading in terms of proximity. Non-clustering nodes send their data to cluster heads. Clustering nodes need greater energy, as compared with typical nodes. Therefore, the fixed cluster head choice wastes energy very soon. LEACH cluster head energy balance is made by a periodical turn of cluster heading among different nodes [7]. Hybrid Energy-Efficient Distributed clustering (HEED protocol) are the other wellknown protocols for WSN clustering. This protocol periodically chooses cluster heads with regard to a combination of residual energy and other parameters including distances between cluster head and its members or order of cluster heads. This algorithm has not any assumption about distribution or density of nodes and their capacities [8]. HEED protocol is different from LEACH in cluster head selection method.

Weighted Clustering Algorithm makes decisions on the basis of weighted set optimization of multiple parameters and a combinational viewpoint, which sometimes needs a large number of comparisons and its efficiency, is impaired [10]. In [11], an algorithm is offered for selecting cluster head nodes by using theory of games. This algorithm acts properly in static distributed WSNs. In the other words, this algorithm cannot put in a good performance for energy consumption optimization in dynamic networks.

Fuzzy Logic Cluster Formation Protocol (FLCFP) is a fuzzy logic algorithm for optimizing energy consumption in wireless sensor networks [6]. This algorithm uses fuzzy inferential system in the cluster formation process. In Particle swarm optimization (PSO), clusters are formed in a way that the consumed energy of the network is distributed within a balanced framework and therefore the energy consumption is reduced and its life is prolonged [12]. Fuzzy energy-aware 
unequal clustering algorithm (EAUCF) attempts to choose nodes as cluster heads that can optimize energy consumption, by using fuzzy relations, the residual energy of the nodes, and distance between nodes [13]. This algorithm uses unequal clustering and - unlike LEACH- forms clusters on the basis of fuzzy relations. The Bayesian-game-based algorithm models energy optimization problem in WSNs in an agreement with Bayesian game and determines cluster head distribution manners on the basis of the residual energy of sensors [14]. According to the findings, this method can distribute network loads in a balanced way, conserve energy and prolong the network life.

\section{THE NETWORK MODEL}

In general, the following properties are given in this study:

- Nodes are limited in terms of energy;

- The related nodes have identical energy and capacity;

- Each node senses the data frequently and always have data for transmission;

- $\quad$ The system is one-staged;

- The nodes can measure distances on the basis of the received signal power; and

- The basic station has an awareness of all sensor data.

\section{THE PROPOSED METHOD}

This section discusses the proposed method, which includes game theory and fuzzy logic for the energy consumption reduction in Wireless Sensor Networks (WSNs).

\subsection{A Determination of Candidate cluster Head Nodes Using Game Theory}

Each network node has two options i.e. it can or cannot act as cluster head. Since the nodes display a selfish behavior, they do not tend to act as cluster head and assign head clustering to other nodes. In the case that none of nodes acts as cluster head, they cannot send their data to the main station and they cannot gain any utility. The best manner of each node occurs when the node itself is not chosen for cluster heading but at least one of the other residual nodes is introduced as cluster heading. Each node utility gained from the data transmission and cluster heading cost are shown respectively by $\mathrm{V}$ and $\mathrm{C}$. In this case, a two-node network is defined, which shows extent of each node utility per the choice of the node itself and the other node (Table 1). Table 1 can be generalized to multiple-node networks. If we assume that D and ND stands respectively for the node selecting itself for cluster heading and the node not being regarded the cluster head, then the node is defined on the basis of interaction between the two nodes (table 1).

Table 1: Utility of Interaction between two nodes in a Two-node Network

\begin{tabular}{|c|c|c|}
\hline & ND & D \\
\hline ND & $(0,0)$ & $(\mathrm{v}, \mathrm{v}-\mathrm{c})$ \\
\hline $\mathrm{D}$ & $(\mathrm{v}-\mathrm{c}, \mathrm{v})$ & $(\mathrm{v}-\mathrm{c}, \mathrm{v}-\mathrm{c})$ \\
\hline
\end{tabular}

Accordingly, If $S=\{D, N D\}$ ( $\mathrm{S}=$ Selection of node i from $\mathrm{S}$ Set), then utility function of each node is defined by Equ. (1):

$U_{i}(s)=\left\{\begin{array}{lr}0 & \text { if } s_{j}=N D \\ v-c & \text { if } s_{i}=D \\ v & \text { if } s_{i}=N D \text { and } s_{j}=D\end{array}\right.$

Therefore, the best manner occurrs when one node is the cluster head and the other nodes are not cluster heads. In this method, each node randomly makes selections in conformity with probability distribution. In the other words, each node has one probability for cluster heading $(\mathrm{P})$ and one probability for non-cluster-heading ( $q=1-p)$. Utility function value for the latter is estimated on the basis of Equ. (2):

$\mathrm{U}_{\mathrm{ND}}=\mathrm{v} \cdot\left(1-(1-\mathrm{p})^{\mathrm{N}-1}\right)$

The expected utility of non-cluster-heading is regarded equal to cluster heading utility ( $\mathrm{v}-\mathrm{c})$ and $\mathrm{P}$ value is measured by Equ. (4):

$\mathrm{v}-\mathrm{c}=\mathrm{v} \cdot\left(1-(1-\mathrm{p})^{\mathrm{N}-1}\right)$

$\mathrm{p}=1-\left(\frac{\mathrm{c}}{\mathrm{v}}\right)^{1 /(\mathrm{N}-1)}$

Then, $\frac{c}{v}$ (a constant number) is regarded equal to W. Equ. (5) is calculated as follows:

$\mathrm{p}=1-\mathrm{w}^{1 /(\mathrm{N}-1)}$

$\mathrm{W}$ is a positive number less than 1 . Accordingly, the $\mathrm{P}$ probability is always between 0 and 1 . By an increase of nodes, $\mathrm{P}$ value is reduced. Conversely, by a reduction of nodes, $\mathrm{P}$ value is increased. By an increase of nodes, they hardly tend to participate in the network because they show selfish behavior. Therefore, $\mathrm{P}$ probability of each node is calculated by measuting $\mathrm{C}$ and $\mathrm{V}$ and determining the energy modell in ordinary nodes and cluster head nodes. Equa. (6) calculates each node energy consumption in case of data transmission. $\mathrm{K}$ and $\mathrm{d}$ are numbers of the sent bits and the distance between node $\mathrm{i}$ and the cluster head respectively. Hence, each node consumes $E_{t x}(k, d)$ in a distance from d for sending the data bits to the cluster head. Additionally, each node's consumed energy for receiving data is calculated by Equa. (7) (each node consumes Erx (k) for receiving the data bit K):

$\mathrm{E}_{\mathrm{tX}}\left(\mathrm{i}, \mathrm{CH}_{\mathrm{i}}\right)=\mathrm{k} \times\left(\mathrm{E}_{\mathrm{elec}}+\varepsilon_{\mathrm{amp}} \times \mathrm{d}_{\mathrm{i}}^{2}\right)$

$\mathrm{E}_{\mathrm{rX}}(\mathrm{k})=\mathrm{E}_{\mathrm{elec}} \times \mathrm{k}$

$E_{\text {elec }}$ and $\varepsilon_{\text {amp }}$ are predefined constants, which are used for clarifying internal circuits of sensors for sending and receiving data as well as other energy consumption operations. When the data are sent from ordinary nodes to cluster heads, the latter aggregate the received data and send them to the main station. If $N_{u}$ is the number of packages which have equal height $(\mathrm{k})$ and are received by the cluster head, then the energy consumed for aggregation of packages is measured by Equa. (8):

Eaggr $=\mathrm{Nu} \times \mathrm{k} \times$ efuse

$e_{\text {fuse }}$ is a constant used for data aggregation. Moreover, the energy consumed for sending data from cluster heads to the 
main station is calculated by Equa. (9) $\left(d_{i}\right.$ is the distance between the cluster head $i$ and the main station):

$\mathrm{E}_{\mathrm{tX}}\left(\mathrm{CH}_{\mathrm{i}}\right.$, Sink $)=\mathrm{k} \times\left(\mathrm{E}_{\text {elec }}+\varepsilon_{\mathrm{amp}} \times \mathrm{d}_{\mathrm{i}}^{4}\right)$

With regard to the above equations, when a node sends its data to the cluster head, it consumes $\mathrm{E}_{\mathrm{tx}}\left(\mathrm{i}, \mathrm{CH}_{\mathrm{i}}\right)$. The cluster headreceives the sent data from the member nodes and sends them to the main station after their aggregation. Hence, the cluster head node cost (c) is estimated by Equa.(10):

$$
\begin{aligned}
\mathrm{E}_{\mathrm{tX}}\left(\mathrm{CH}_{\mathrm{i}}, \mathrm{Sink}\right) \times \mathrm{c}=\mathrm{N}_{\mathrm{u}} \times \mathrm{E}_{\mathrm{rX}} \times \mathrm{E}_{\text {aggr }} \\
\mathrm{c}>\mathrm{E}_{\mathrm{tX}}\left(\mathrm{i}, \mathrm{CH}_{\mathrm{i}}\right)=\mathrm{t}
\end{aligned}
$$

If $\alpha$ and $\beta$ are data transmission rate and data receipt rate respectively, then the exchanged energy for each node $\left(E_{D}\right)$ is calculated by Equa. (11). The resulted utility (v) is measured by Equa. (12):

$\mathrm{E}_{\mathrm{D}}=\alpha \cdot \mathrm{E}_{\mathrm{tX}}\left(\mathrm{i}, \mathrm{CH}_{\mathrm{i}}\right)+\beta \cdot \mathrm{E}_{\mathrm{rX}}$

$\mathrm{v}=(\alpha+\beta) \times \mathrm{E}_{\mathrm{tx}}-\mathrm{ED}$

Accordingly, the probability of node cluster heading (p) is measured as follows:

$\mathrm{p}=1-\left(\frac{\mathrm{c}-\mathrm{t}}{\mathrm{v}-\mathrm{t}}\right)^{1 /(1-\mathrm{N})}$

\subsection{Cluster Head Member Selection Using Fuzzy Logic}

The fuzzy logic is used for an optimal allocation of members to cluster heads. As a number of nodes should be allocated to cluster heads, three fuzzy criteria is used:

- The distance between the node and each cluster head;

- Residual energy of cluster heads; and

- The distance between cluster heads and the main station.

The distance between the node and each cluster head is fuzzed in terms of near distance, middle distance, and far distance. The fuzzy value of this distance is measured by Equa. (14):

Value_Distance_node $_{i}=\frac{\text { Distance_node }_{i}}{\text { Max (Distance_nodes })}$

Value Distance node $e_{i}$ is the distance of node $\mathrm{i}$ from the node having the greatest distance from the cluster head. Max(Distance nodes) an Distance node respectively show the node having the greatest distance from the cluster head and the distance of node i. After each node distance from the cluster head is calculated, it can be categorized as near distance, middle distance, and far distance. As a result, these values are calculated on the basis of fuzzy input variables (Fig 1).

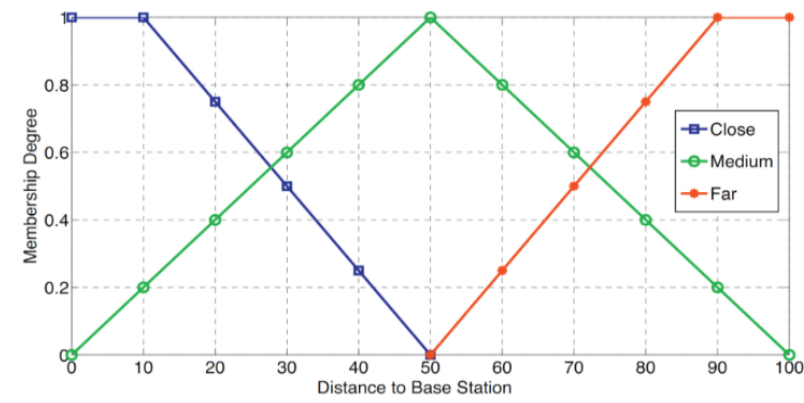

Fig 1: Fuzzy Input Variables of Each Node Distance to Cluster Heads

For fuzzing residual energy of cluster heads, the fuzzy numbers of each node's residual energy are considered as follows: In each turn, the cluster heads having greatest residual energy are identified and the fuzzy numbers are considered between 0 and 1. Consequently, fuzzy roles of network energy and cluster heads' residual energy are changed. Fuzzy input variables are changed in each turn in terms of the residual energy i.e. fuzzy input variables are defined in each turn in an agreement with cluster heads' residual energy. Fig 2 shows fuzzy input variables of cluster heads' residual energy.

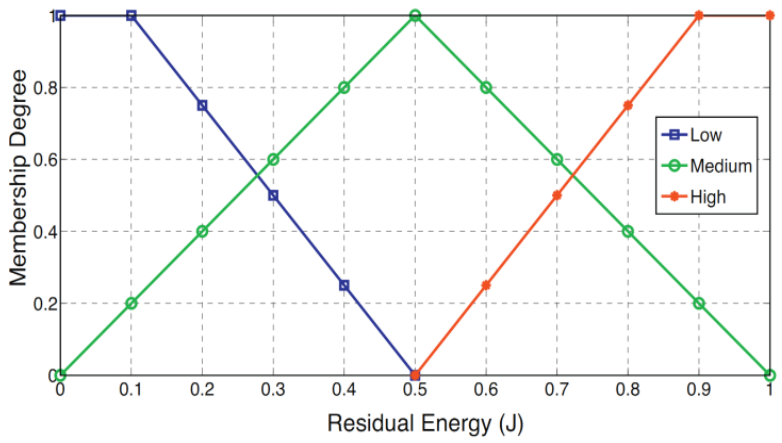

Fig 2: Fuzzy Input Variables of Cluster Heads' Residual Energy

For fuzzing each cluster head's distance to the main station, the cluster heads' distance to the main station is defined in fuzzy terms. Same as two other parameters, near, middle, and far distances are defined.

According to the above-mentioned properties, fuzzy rules for cluster heads' member allocation is defined on the basis of Table 2. A membership opportunity is defined for each node, by which cluster head-node allocations are determined. With regard to each node's membership opportunity, probability values are measured for each level of membership opportunity (Table 3). The proper cluster head is selected by employing Roulette Wheel Selection (RWS) (a selection method employed for genetics algorithm). 
Table 2: Probability for Each Level

\begin{tabular}{|c|c|c|c|c|c|c|c|c|c|}
\hline 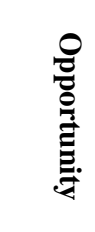 & 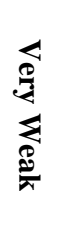 & 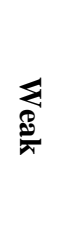 & 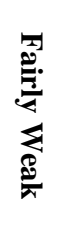 & 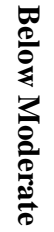 & $\begin{array}{l}\frac{3}{2} \\
\frac{0}{9} \\
\frac{0}{0}\end{array}$ & 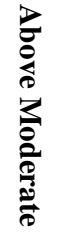 & 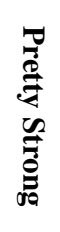 & 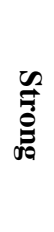 & 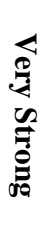 \\
\hline $\begin{array}{c}\text { Proba } \\
\text { bility }\end{array}$ & 0.1 & 0.2 & 0.3 & 0.4 & 0.5 & 0.6 & 0.7 & 0.8 & 9 \\
\hline
\end{tabular}

Table3: If-Then Rules for Cluster Heads' Member Allocation

\begin{tabular}{|c|c|c|c|}
\hline $\begin{array}{l}\text { Membership } \\
\text { Probability }\end{array}$ & 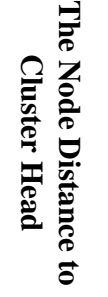 & 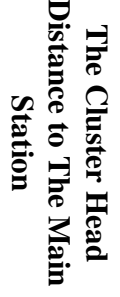 & 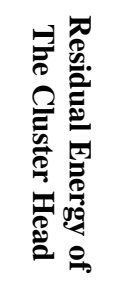 \\
\hline Very Weak & Far & Far & Low \\
\hline Weak & Far & Far & Average \\
\hline Fairly Weak & Far & Far & High \\
\hline Weak & Far & Middle & Low \\
\hline Fairly Weak & Far & Middle & Average \\
\hline $\begin{array}{l}\text { Below } \\
\text { Moderate }\end{array}$ & Far & Middle & High \\
\hline Fairly Weak & Far & Near & Low \\
\hline $\begin{array}{l}\text { Below } \\
\text { Moderate }\end{array}$ & Far & Near & Average \\
\hline Moderate & Far & Near & High \\
\hline Fairly Weak & Middle & Far & Low \\
\hline $\begin{array}{l}\text { Below } \\
\text { Moderate }\end{array}$ & Middle & Far & Average \\
\hline Moderate & Middle & Far & High \\
\hline $\begin{array}{l}\text { Below } \\
\text { Moderate }\end{array}$ & Middle & Middle & Low \\
\hline Moderate & Middle & Middle & Average \\
\hline \begin{tabular}{|l|} 
Above \\
Moderate
\end{tabular} & Middle & Middle & High \\
\hline Moderate & Middle & Near & Low \\
\hline $\begin{array}{l}\text { Above } \\
\text { Moderate }\end{array}$ & Middle & Near & Average \\
\hline Fairly Strong & Middle & Near & High \\
\hline Moderate & Near & Far & Low \\
\hline $\begin{array}{l}\text { Above } \\
\text { Moderate }\end{array}$ & Near & Far & Average \\
\hline Fairly Strong & Near & Far & High \\
\hline $\begin{array}{l}\text { Above } \\
\text { Moderate }\end{array}$ & Near & Middle & Low \\
\hline Fairly Strong & Near & Middle & Average \\
\hline Strong & Near & Middle & High \\
\hline Fairly Strong & Near & Near & Low \\
\hline Strong & Near & Near & Average \\
\hline Very Strong & Near & Near & High \\
\hline
\end{tabular}

This Study employs Time Division Multiple Access (TDMA) for avoiding data transmission inference. Each cluster node is attributed to a time period for data transmission. The cluster head plans the schedule by defining properties to all cluster members. Accordingly, each node sends the sensed data to the cluster head in the allocated time period and the cluster head in turn sends them to the main station. After data transmission, clustering operation are started again (the first phase) and clusters are formed again. Phases 1 and 2 continue by the end of WSN life. Fig 3 shows the proposed algorithm stages.

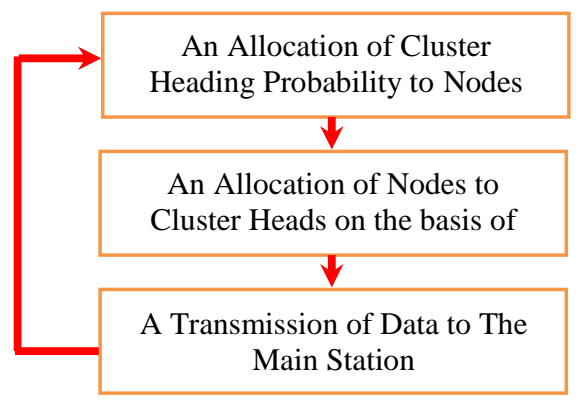

Figure3: The Proposed Algorithm Stages

\section{AN EVALUATION OF SIMULATION RESULTS}

MATLAB is used for testing and validating the proposed algorithm. For a careful comparison of results, different nodes (three different scenarios) are utilized. Table 4 shows different scenarios for evaluating the simulation results. Values of parameters and the considered conditions are based on [2] and [16].

Table 4: The Simulation Parameters

\begin{tabular}{|l|c|c|c|}
\hline Parameter & Scenario 1 & Scenario 2 & Scenario 3 \\
\hline $\begin{array}{l}\text { Numbers of } \\
\text { Nodes }\end{array}$ & 50 & 100 & 200 \\
\hline Network Size & $100 \times 100$ \\
\hline $\begin{array}{l}\text { Main Station } \\
\text { Location }\end{array}$ & $(100,50)$ \\
\hline $\begin{array}{l}\text { Primary } \\
\text { Each Node }\end{array}$ & 100 Joule \\
\hline$\varepsilon_{\text {amp }}$ & \multicolumn{2}{|c|}{0.0001 Joule } \\
\hline$E_{\text {elec }}$ & \multicolumn{2}{|c|}{0.00005 Joule } \\
\hline
\end{tabular}

The proposed algorithm is compared and contrasted with EAUCF and LEACH. The simulations are evaluated in terms of live nodes, the first node death period, and the last node death period.

Fig 4 shows scenario 1 results including numbers of live nodes and their death in different periods. The node death in the proposed algorithm occurs later, as compared with EAUCF and LEACH. Accordingly, the proposed algorithm puts in a better performance relative to the other two protocols. Also, the last network node is destroyed in the 
proposed algorithm later as compared with the other two protocols. According to results, the proposed algorithm can prolong the network life in the first scenario. Fig 5 shows scenario 2 results. The node death rate is limited in the proposed algorithm relative to EAUCF and LEACH.

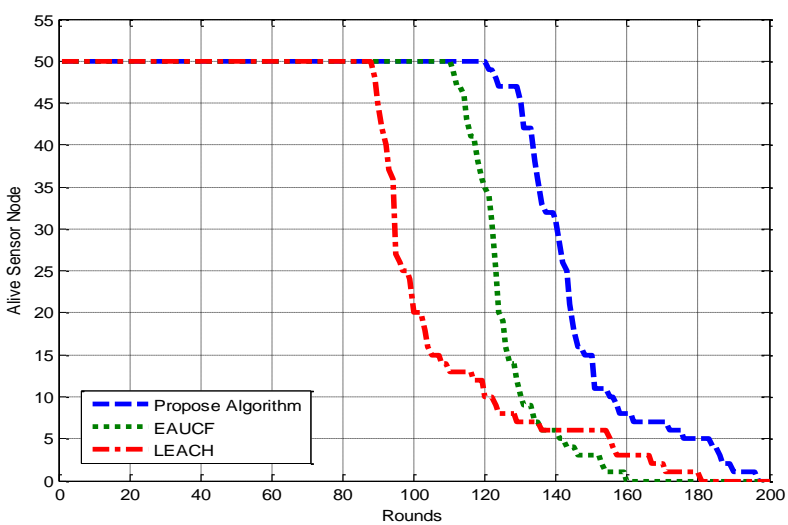

Figure4:Live Node Distribution in Terms of period numbers in Scenario 1

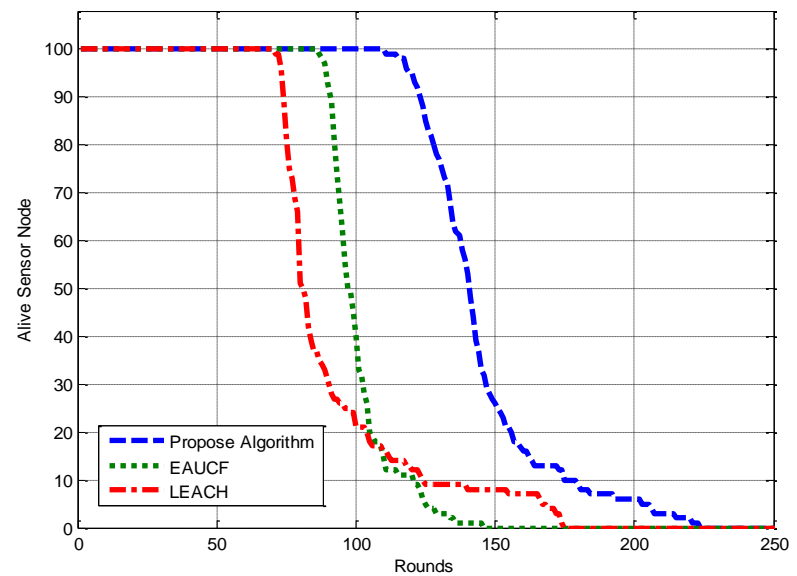

Figure5: Live Node Distribution in Terms of period numbers in Scenario 2

Fig 6 shows death of the network nodes in scenario 3. In this scenario, the proposed algorithm can prolong the network life as compared with EAUCF and LEACH. The node death occur more slowly and therefore, the proposed algorithm can reduce energy consumption.

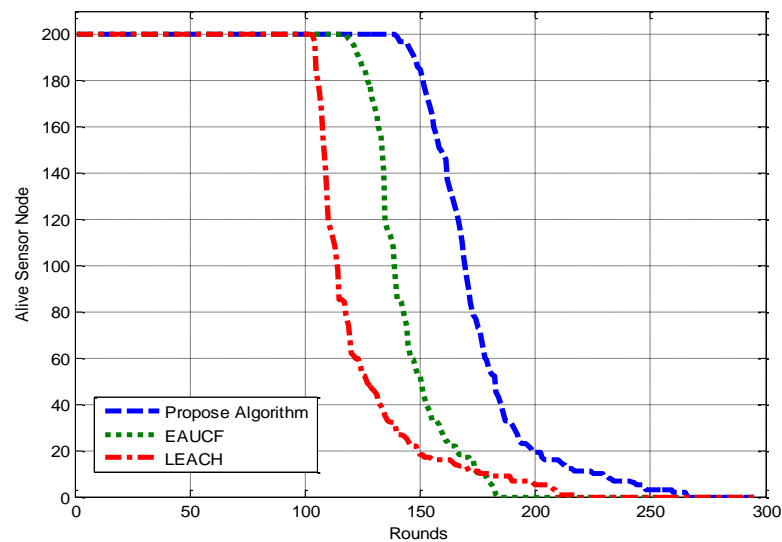

Figure6: Live Node Distribution in Terms of period numbers in Scenario 3
Fig 7 shows the first node death and the last node death in scenario 1. FND and LND are the first node dead and the last node dead respectively. In scenario 1, the proposed algorithm could delay the first and the last node death time. Therefore, the proposed algorithm can prolong the network life as compared with EAUCF and LEACH and give a complete coverage. This method can prolong the network life by $\% 10$ and $\% 27$ respectively relative to EAUCF and LEACH. In Scenario 1 , the method can put in a good performance.

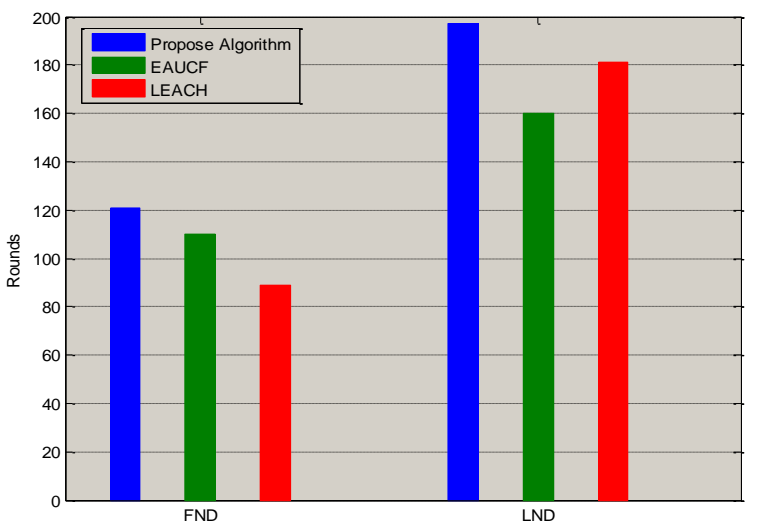

Fig 7: FND \& LND Results in Scenario 1

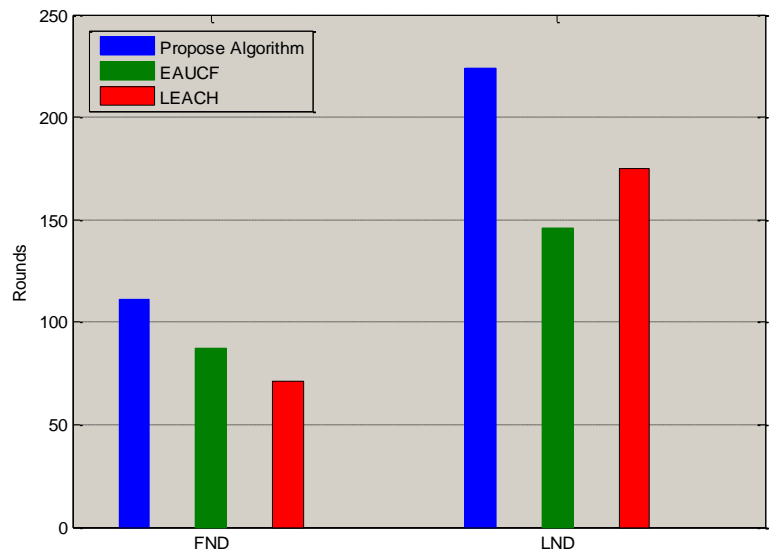

Fig 8: FND \& LND Results in Scenario 2

Fig 8 shows First Node Dead(FND) and Last Node Dead (LND) in Scenario 2. The proposed algorithm can delay the first and the last node death time. Similarly, it could put in a good performance in scenario 3 in terms of FND and LND as compared with EAUCF and LEACH (Fig 9). It is worth noting that FND time is more crucial in WSNs i.e. the more the FND is delayed, the more complete the node can cover its concerned environment. Hence, the shorter the interval between FND and LND is, the more balanced the algorithm can distribute energy consumption among nodes. Therefore, FND is delayed and the network can cover its environment in a longer period of time and more effectively. As simulation results suggest, the proposed algorithm can distribute the consumed energy in a balanced way as compared with the other two algorithms. 


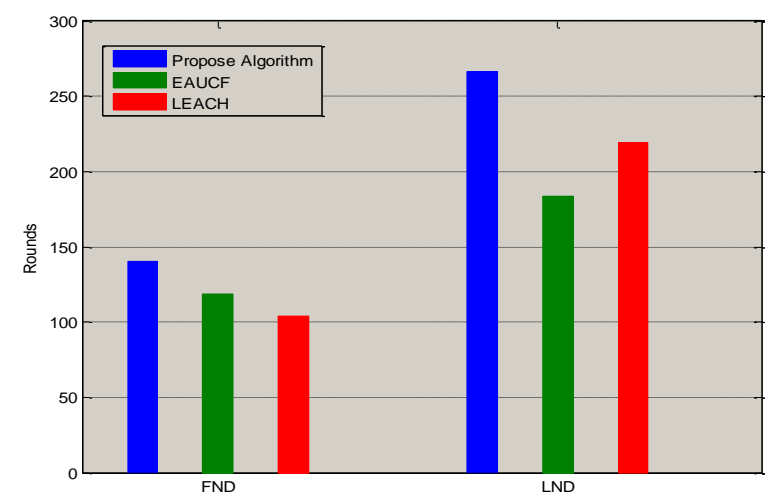

Fig 9: FND \& LND Results in Scenario 3

\section{CONCLUSION}

One of WSN problems is energy resource limitations. This study introduces a new method on the basis of game theory and fuzzy logic. The simulation findings reveal that the proposed algorithm can put in a more proper performance and prolong the network life as compared with similar algorithms. In the proposed method, the basic station is static. Therefore, future studies can concentrate on dynamicity of basic station. Moreover, the proposed method is a centralized protocol in which clusters are formed by the basic station. Future studies can design distributed-environment-related algorithms.

\section{REFERENCES}

[1] ZhixinLiua, QingchaoZhenga, LiangXuea, XinpingGuan. A distributed energy-efficient clustering algorithm with improved coveragein wireless sensor networks. Future Generation Computer Systems. 2012. 28:780-790.

[2] Zhu Yong, Qing Pei. A Energy-Efficient Clustering Routing Algorithm Based on Distance and Residual Energy for Wireless Sensor Networks. International Workshop on Information and Electronics Engineering. 2012. 29:1882-1888.

[3] PratyayKuila, Prasanta K. Jan. Energy Efficient LoadBalanced Clustering Algorithm for Wireless Sensor Networks. International Conference on Communication, Computing \& Security. 2012. 7:771-777.

[4] RogaiaMhemed ,NaumanAslam, William Phillips and Frank Comeau. An Energy Efficient Fuzzy Logic Cluster Formation Protocol in Wireless Sensor Networks. International Conference on Ambient Systems, Networks and Technologies. 2012. 10:255-262.

[5] $\mathrm{Hu} \mathrm{Yu,} \mathrm{Wang} \mathrm{Xiaohu.} \mathrm{PSO-based} \mathrm{Energy-balanced}$ Double Cluster-heads Clustering Routing for wireless sensor networks. Advanced in Control Engineering and Information Science. 2011. 15:3073 - 3077.

[6] WANG Yan, SHAN Xin-xin, SUN Yanming. Study on the application of Genetic Algorithms in the optimization of wireless network. International Workshop on Automobile, Power and Energy Engineering. 16 (2011) $348-355$.

[7] Ashutosh Kumar Singh, Sandeep Goutele, S.Verma and N. Purohit. An Energy Efficient Approach for Clustering in WSN using Fuzzy Logic. International Journal of Computer Applications (0975 - 8887). Volume 44No18, April 2012.

[8] RogaiaMhemed, NaumanAslam, William Phillips and Frank Comeau. An Energy Efficient Fuzzy Logic Cluster Formation Protocol in Wireless Sensor Networks. International Conference on Ambient Systems, Networks and Technologies. 10 (2012) 255 - 262.

[9] Khalid A. Darabkh, Shereen S. Ismail, Mohammad AlShurman, Iyad F. Jafar, EmanAlkhader, Mamoun F. AlMistarihi. Performa nce evaluation of selective and adaptive heads clustering algorithms over wireless sensor networks. Journal of Network and Computer Applications 35 (2012) 2068 -2080.

[10] Buddha Singh, D. K. Lobiyal. Energy-aware cluster head selection using particle swarm optimization and analysis of packet retransmissions in WSN. C3IT-2012. 4 (2012) $171-176$.

[11] Chengfa Li, Mao Ye, Guihai Chen. An Energy-Efficient Unequal Clustering Mechanism for Wireless Sensor Networks, Mobile Adhoc and Sensor Systems Conference, 2005. IEEE International Conference on. 77 Nov. 2005.

[12] Zhu Yong, Qing Pei. An Energy-Efficient Clustering Routing Algorithm Based on Distance and Residual Energy for Wireless Sensor Networks. International Workshop on Information and Electronics Engineering (IWIEE). 29 (2012) $1882-1888$.

[13] Buddha Singh and DayaKrishanLobiyal. A novel energy-aware cluster head selection based on particle swarm optimization for wireless sensor networks. Singh and Lobiyal Human-centric Computing and Information Sciences. 2012.

[14] SajidHussain, Abdul W. Matin and Obidul Islam. Genetic Algorithm for Energy Efficient Clusters in Wireless Sensor Networks. Information Technology, 2007. ITNG '07. Fourth International Conference on. 2-4 April 2007.

[15] Atashpaz-Gargari. Imperialist competitive algorithm: An algorithm for optimization inspired by imperialistic competition. Evolutionary Computation, 2007. CEC 2007. IEEE Congress on. 25-28 Sept. 2007.

[16] HakanBagci, Adnan Yazici. An energy aware fuzzy approach to unequal clustering in wireless sensor networks. Applied Soft Computing. 13 (2013) 17411749.

[17] SajidHussain, Abdul W. Matin and Obidul Islam. Genetic Algorithm for Energy Efficient Clusters in Wireless Sensor Networks. Information Technology, 2007. ITNG '07. Fourth International Conference on. 2-4 April 2007.

[18] Georgios Koltsidas, Fotini-Niovi Pavlidou. A Game Theoretical Approach to Clustering of Ad-Hoc and Sensor Networks. Telecommunication Systems. June 2011, Volume 47, Issue 1-2, pp 81-93. 